\title{
A comprehensive, multispecialty approach to an acute exacerbation of chronic central pain in a tetraplegic
}

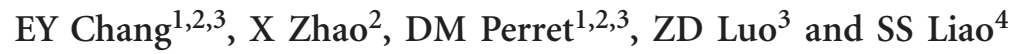

\begin{abstract}
Study design: We present a case report describing the multidisciplinary treatment of a tetraplegic spinal cord injury (SCI) patient who developed an acute exacerbation of chronic central pain.

Objective: To bring further awareness to the importance of using a comprehensive, multidisciplinary approach in treating acute exacerbation of chronic central pain in $\mathrm{SCl}$ patients.

Setting: University of California Irvine Medical Center, Orange, CA, USA.

Case report: We present a 34-year-old man with a past medical history of C5 American Spinal Injury Association B tetraplegia secondary to a surfing accident 8 years prior, central pain syndrome, spasticity, autonomic dysreflexia and anxiety who arrived at the emergency room with a 1-month history of worsening acute on chronic pain refractory to opioid escalation. The multispecialty treatment plan included treatment of the patient's urinary tract infection by the primary medicine service, management of the patient's depression by the psychiatric service, treatment of bowel obstruction by general surgery and adjustment of pain medications by pain management. The patient was found to have stable neurological findings, neuroimaging unchanged from prior imaging and a urinary tract infection. Hospitalization was complicated by severe colonic dilation that required disimpaction by general surgery.

Conclusion: The treatment of this patient's acutely worsened central pain highlights the importance of applying a multidisciplinary approach to $\mathrm{SCl}$ patients with an acute exacerbation of chronic central pain. In this case, the multispecialty treatment plan included treatment of the patient's urinary tract infection by the primary medicine service, management of the patient's depression by the psychiatric service, treatment of bowel obstruction by general surgery, and adjustment of pain medications by pain management.
\end{abstract} Spinal Cord (2014) 52, S17-S18; doi:10.1038/sc.2014.12; published online 11 February 2014

Keywords: spinal cord injury; central pain; multidisciplinary; acute on chronic; case report; tetraplegia

\section{INTRODUCTION}

Spinal cord injury (SCI) is a devastating injury that affects the motor and sensory function of patients. These patients developed pain $69-86 \%$ of the time following the initial injury and up to one-third described the pain as severe. ${ }^{1}$ In these patients, pain results in a marked decrease in quality of life and negatively affects participation in activities of daily living. ${ }^{2}$ Mechanistically, SCI results in the dissociation of the supraspinal control centers from the peripheral and autonomic nervous systems and also interrupts the homeostasis between the facilitatory and inhibitory systems at the spinal cord level below the level of injury. This may lead to an increase in the patient's pain state via sympathetic efferent activity, humoral sympathetic mediation, ephaptic interaction or alpha-adrenergic receptor hypersensitivity. ${ }^{3}$ We would like to bring further awareness to the importance of using a comprehensive, multidisciplinary approach in treating acute on chronic central pain in SCI patients by presenting a specific case.

\section{CASE REPORT}

We present a 34-year-old man with a history of C5 American Spinal Injury Association B tetraplegia secondary to a surfing accident 8 years prior, who arrived at the emergency room with a 1-month history of acutely worsening pain refractory to opioid escalation. The patient had a past history of central pain syndrome, spasticity, autonomic dysreflexia and anxiety that had been controlled on an outpatient regimen of methadone, sustained-release oxycodone, fentanyl patch, pregabalin, duloxetine, baclofen, tizanidine and trazodone. The patient reported that his anxiety, depression, diaphoresis and blood pressure had become extremely difficult to control in the past month, and that the increasing pain had caused him to have suicidal ideations. The patient's pain had increased from a baseline of 5 out of 10 on Visual Analog Scale (VAS) to ' 14 out of 10' despite increases in methadone dosage and having started on ketamine. The patient described his pain as either 'dry ice on his skin' or 'being dunked in hot water from the nipples on down'.

Physical examination revealed only stable neurological findings consistent with the prior SCI. Laboratory examination revealed normal chemistry and a urinary tract infection that was treated with intravenous antibiotics. Neuroimaging of the spine showed no changes compared with previous imaging. Psychiatrist was consulted for his suicidal ideations, and amitriptyline, olanzapine and valproate were started. Pain management was consulted and recommended discontinuing all home medications except ketamine with the addition of diazepam and clonidine. On hospital day 2, the patient

${ }^{1}$ Division of Pain Medicine, Department of Anesthesiology \& Perioperative Care, The Center for Pain Medicine, Irvine, CA, USA; ${ }^{2}$ Department of Physical Medicine \& Rehabilitation, University of California Irvine Medical Center, Orange, CA, USA; ${ }^{3}$ Department of Anesthesiology \& Perioperative Care, University of California Irvine Medical Center, Orange, CA, USA and ${ }^{4}$ Department of Palliative Medicine, University of California Irvine Medical Center, Irvine, CA, USA

Correspondence: Dr EY Chang, Department of Physical Medicine \& Rehabilitation, University of California Irvine Medical Center, Orange, CA 92697, USA.

E-mail: changey1@uci.edu

Received 21 October 2013; accepted 13 January 2014; published online 11 February 2014 
developed severe abdominal distention and colonic dilation that were refractory to medical management and required disimpaction under general anesthesia by general surgery. After initial interventions, the patient reported that his pain decreased to 3 out of 10 on the VAS, and patient felt well enough to be discharged from the hospital. A follow-up visit 2 months later showed no return of pain exacerbations beyond the patient's baseline pain.

\section{DISCUSSION}

Central neuropathic pain in SCI patients has been an understudied area in research. SCIs are typically followed by anatomical changes, neurochemical changes and inflammatory changes that result in damage to the central nervous system. These chemical changes may result in the release of excitatory amino acids as well as the production of free radicals that cause further damage. These events create a scenario where sensory neurons of the spinal cord are affected and result in hyperalgesia and allodynia. ${ }^{4}$ In addition, anxiety and mood disorders such as depression may develop in SCI patients, which in turn elevate the sympathetic nervous system activity. In patients with major depressive disorder, higher central nervous nuclei mediating the affective, autonomic or cognitive components of the activation of the neural network of pain perception are believed to be maladaptive. Dysfunction in the autonomic nervous system occurs in patients with central pain states, as evidenced by many of these patients having symptoms of orthostatic intolerance. ${ }^{5}$
We believe that the best way to manage an acute exacerbation of chronic SCI central pain is to use a multifaceted approach. In our case presentation, resolving the patient's pain required treatment of his urinary tract infection by the primary medicine service, management of his depression by the psychiatric service, treatment of his bowel obstruction by general surgery and adjustment of his pain medications by pain management.

\section{CONFLICT OF INTEREST}

The authors declare no conflict of interest.

\section{ACKNOWLEDGEMENTS}

I would like to acknowledge the support in part by NIH grants: K12 HD001097 and UL1 TR000153 (EYC).

1 Casey KL. Pain and Central Nervous System Disease: The Central Pain Syndromes. Raven Press: New York, NY, USA, 1991.

2 Murray RF, Asghari A, Egorov DD, Rutkowski SB, Siddall PJ, Soden RJ et al. Impact of spinal cord injury on self perceived pre- and postmorbid cognitive, emotional and physical functioning. Spinal Cord 2007; 45: 429-436.

3 McMahon SB. Mechanisms of sympathetic pain. Br Med Bull 1991; 47: 584-600.

4 Teasell RW, Mehta S, Aubut JA, Foulon B, Wolfe DL, Hsieh JT et al. A systematic review of pharmacologic treatments of pain after spinal cord injury. Arch Phys Med Rehabil 2010; 91: 816-831.

5 Furlan R, Jacob G, Snell M, Robertson D, Porta A, Harris P et al. Chronic orthostatic intolerance: a disorder with discordant cardiac and vascular sympathetic control. Circulation 1998; 98: 2154-2159. 